\title{
Criminologie
}

\section{La délinquance d'hier et de demain au Québec}

\section{Marc LeBlanc}

Volume 8, numéro 1-2, 1975

Délinquance juvénile au Québec

URI : https://id.erudit.org/iderudit/017042ar

DOI : https://doi.org/10.7202/017042ar

Aller au sommaire du numéro

Éditeur(s)

Les Presses de l'Université de Montréal

ISSN

0316-0041 (imprimé)

1492-1367 (numérique)

Découvrir la revue

Citer cet article

LeBlanc, M. (1975). La délinquance d'hier et de demain au Québec.

Criminologie, 8(1-2), 145-158. https://doi.org/10.7202/017042ar d'utilisation que vous pouvez consulter en ligne.

https://apropos.erudit.org/fr/usagers/politique-dutilisation/ 
LA DÉLINQUANCE D'HIER ET DE DEMAIN AU QUÉBEC

Marc LeBlanc 
La délinquance d'hier et de demain, voilà un titre facile et ambitieux. Facile parce que la délinquance d'hier s'observe dans les rapports statistiques et ambitieux parce que l'homme a toujours tenté de prévoir l'avenir mais sans beaucoup de succès.

Dans une première section, nous décrirons la délinquance juvénile, ses grandes lignes d'évolution sur les plans du volume des infractions connues. Dans une seconde section, nous extrapolerons les tendances observées de manière à prévoir la délinquance de demain.

\section{A. LA DELINQUANCE D'HIER}

La police est la première agence de régulation sociale qui puise dans le réservoir des infractions commises par les juvéniles ; elle sanctionne, par l'arrestation, les comportements qui violent les codes et règlements, plus particulièrement le code criminel.

L'évolution de la délinquance sera décrite à l'aide des taux d'arrestations entre 1932 et 1972 . Les chiffres bruts de la délinquance ont été tirés du rapport annuel de la police de Montréal. Ces rapports sont comparables de 1932 à 1962. A partir de 1962, les données ont été extraites de la formule « $C$ » de Statistique Canada. La combinaison de ces deux séries assure des données suffisamment homogènes sur une période de quarante ans. Ces données concernent, plus précisément, les inculpations d'adolescents de 7 à 17 ans, inclusivement, pour des infractions au code criminel, aux règlements municipaux et aux divers statuts et règlements fédéraux et provinciaux. 
La figure 1 présente l'évolution de la délinquance durant la période étudiée. On note d'une façon générale, que le nombre d'arrestations des juvéniles des deux sexes suit à Montréal une trajectoire ascendante. Malgré cette tendance générale à la hausse, il ressort à la figure 1 que la délinquance se situe sur trois paliers : de 1932 au début des années quarante, du début des années quarante au milieu des années soixante et de cette période à maintenant. À l'intérieur de ces paliers, on observe quatre cycles, c'est-à-dire une hausse de la délinquance sur quelques années suivie d'une baisse. Ces cylces sont les suivants : 1932 à 1941, 1941 à 1954, 1954 à 1962 et 1962 à 1972 ; ce sont des cycles d'une dizaine d'années. Ces cycles correspondent à la période de la crise économique, de la seconde guerre mondiale, à la reprise économique de l'après-guerre et à la société d'affluence actuelle.

Bien que les fluctuations économiques et politiques aient une influence sur la délinquance, il n'est pas permis de s'appuyer sur ces effets exogènes pour expliquer toutes les fluctuations de la figure 1. Notamment, il convient d'expliquer le haut niveau de la délinquance en 1943, 1944 et 1945 et l'accroissement très important de la délinquance durant les années soixante. Si ce n'était de ces fluctuations très importantes, la trajectoire de la délinquance serait marquée de cycles assez réguliers. Le haut niveau de délinquance en 1943, 1944 et 1945 s'explique par la hausse de la répression des violations aux règlements municipaux, notamment le couvre-feux et des règlementations de contrôle des biens rares (voir Chevrier et al., 1974). Pour sa part, l'accroissement phénoménal de la délinquance durant les années soixante s'explique par une amélioration dans la cueillette des données (voir Statistique Canada, 1971), par des modifications organisationnelles (davantage de policiers et professionnalisation, voir Chevrier et al., 1974) et une efficacité accrue (Fréchette, 1973, signale que les arrestations ont augmenté plus rapidement que les délits rapportés).

Si l'on pondère cette croissance fictive, l'on obtient un taux de croissance de la délinquance totale qui, tout en étant réel, semble plus normal tout au long de la décade 1960-1970, l'augmentation réelle de la délinquance juvénile à Montréal et aussi au Québec (LeBlanc, 1974) n'a donc pas été alarmante même si elle a été substantielle et de plus elle semble s'être conformée au profil évolutif de la délinquance dans la plupart des autres sociétés 





industrielles (Europe : Christiansen, 1967; Europe de l'Est : Jasinski, 1968 ; Canada : Giffen, 1965 et LeBlanc, 1968 ; EtatsUnis : Gibbons, 1968).

L'augmentation réelle serait alors le fruit de l'expansion considérable des naissances après la deuxième guerre mondiale. Cette vague a amené un surplus de clientèle dans les services de justice à partir des années soixante jusque vers 1969 ; autour de 1970, elle se résorbe de plus en plus (par exemple, on ferme de plus en plus d'écoles et on parle de surplus de professeurs). De plus, il est certain qu'une densité plus grande signifie plus d'occasions, de conflits et d'entraînements mutuels.

Un autre facteur qui explique l'augmentation réelle de la délinquance est l'engagement des jeunes dans de nouvelles formes de déviance. Ainsi, entre 1965 et 1970, le Québec, comme toutes les sociétés occidentales, a subi un bouillonnement socio-culturel (la révolution tranquille) concomittant à une augmentation de l'usage de la drogue, du "hippyisme », des bandes structurées dites de motards, du « drop-out» scolaire et des formes violentes de contestation étudiante. L'effet de l'expansion de ces nouvelles formes de délinquance s'observe dans les statistiques de la délinquance par une diminution de l'importance de la délinquance traditionnelle (notamment le vol) au profit d'autres statuts et règlements plus vagues (notamment les lois provinciales) (LeBlanc, 1974).

À partir de 1970 , le tableau de la délinquance semble se modifier. En premier lieu, la courbe générale de la délinquance est marquée par une trajectoire descendante sinon de stabilisation. En second lieu, les nouvelles formes de délinquance, qui constituaient l'autre source d'augmentation de la délinquance, sont elles aussi en régression: l'usage de la drogue diminue (Delagrave, 1972); les groupements hippies se sont dissous, les gangs de motards ont à peu près disparu (Legendre et al., 1973), la contestation étudiante est calme et le « drop-out 》 scolaire se stabilise (Martineau, 1974).

Si les commentaires précédents nous permettent de mieux comprendre certaines caractéristiques de la trajectoire de la délinquance, il n'en demeure pas moins que la courbe d'évolution de la délinquance, sur une période de temps prolongée, adopte une allure sinusoïdale, avec des sommets qui réapparaissent tous les sept à dix ans, environ. Il semble vraiment que nous soyons en 
présence de cycles ou de phases, ceci malgré une tendance générale à la hausse.

En somme, la délinquance juvénile à Montréal se conforme à la loi de la régularité dynamique de la criminalité de Ferri (1893). Par cette loi, Ferri voulait affirmer que dans son ensemble la criminalité augmente régulièrement si l'on analyse des séries à long terme. Et par ses cycles et phases, la délinquance juvénile se compare aux variations cycliques des autres phénomènes socioculturels que décrit Sorokin (1957). Si ces déterminismes (régularité dynamique et cyclique de l'évolution de la délinquance) tiennent, nous devrions nous attendre à une expansion de la délinquance à la fin des années 1970 et à une résurgence des formes inédites de conduites délinquantes.

\section{B. LA DELINQUANCE DE DEMAIN}

Quel sera le volume de la délinquance en 1975 , en 1980 ? De façon à estimer le volume de la délinquance pour les années à venir, nous avons utilisé la méthode de Box et Jenkins (1971). Cette méthode est résumée en annexe et présentée en détail dans Chevrier et al. (1974). À la figure 2 et au tableau 1, on peut observer le volume prévu de délinquance pour chacune des années.

On note que l'ensemble de la délinquance juvénile sera à la baisse, le taux de délinquance passera de $17,66 \%$ en 1973 à $14,64 \%$ en 1980 . Ainsi la tendance initiée en 1969 se continue et le taux de délinquance diminuera de 3,7\% entre 1972 et 1973. Par ailleurs, en 1974 et 1976, la diminution se continuera mais elle sera de moins en moins marquée. On peut même penser que le volume augmentera à un moment donné, à mesure que le modèle de prévision sera ajusté avec les années qui passent.

Comment expliquer cette baisse prévue de la délinquance ? Plusieurs facteurs sont à retenir notamment une diminution de la densité de la population juvénile et un climat social moins effervescent. La délinquance est indéniablement liée à la densité de la population juvénile (Jepsen et Pal, 1969 ; Chein, 1963) ; puisque la population juvénile diminuera au Québec, il peut donc en découler une diminution de la délinquance. Parallèlement le climat social général est marqué par l'accalmie, le bouillonnement socioculturel des années soixante n'existe plus; il y a bien l'inflation, mais puisqu'elle est inscrite dans l'affluence, elle est alors un tracas 
FIGURE 2

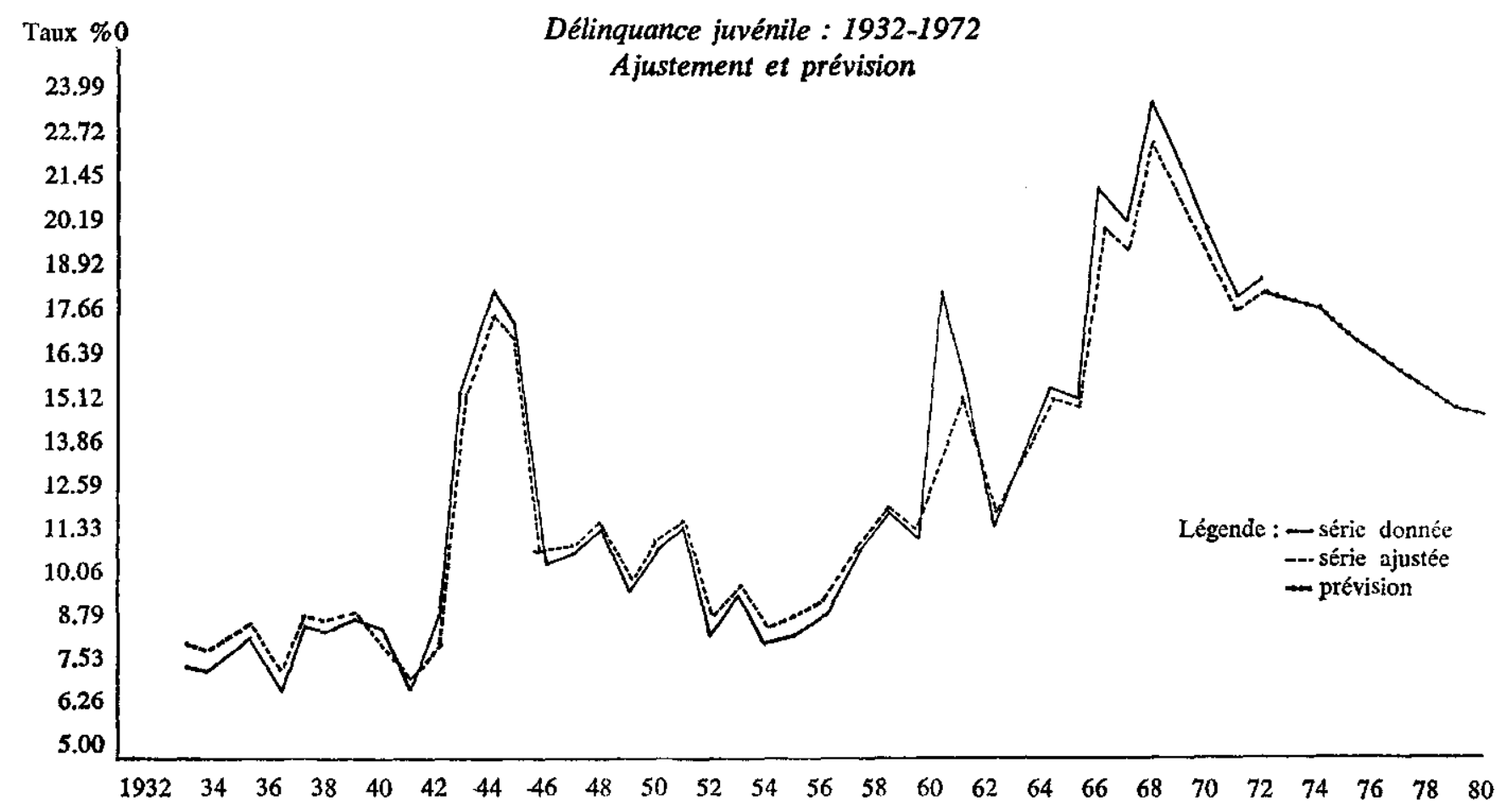


plutôt qu'un problème. Sur le plan politique, les majorités sont stables et on ne parle plus de révolution même tranquille.

En conséquence, nous n'avons pas à craindre, dans l'immédiat tout au moins, l'éclosion de nouvelles formes de délinquance, en sera-t-il toujours ainsi? Pour la fin des années soixante-dix nous sommes portés à croire que certaines formes de déviances réapparaîtront suivant de nouvelles modalités. Cette extrapolation repose sur la constatation que les hausses de la délinquance sont cycliques et il nous apparaît qu'il en est de même avec diverses formes de déviance. Ainsi comme les existentialistes de la fin des années quarante se sont mutés en «beatnik * à la fin des années cinquante, ceux-ci se sont transformés en «hippies > à la fin des années soixante, mais qui les remplacera dans cette décennie? Comme les «zoot suit » de la fin des années quarante se sont continués dans les vestes de cuir de la fin des années cinquante puis en motards dix ans plus tard, y aura-t-il d'autres groupes violents dans cette décennie ou d'autres formes de violence? Pour leur part, l'usage des drogues, l'abandon scolaire et la contestation étudiante trouveront sûrement d'autres modes d'expression.

Au terme de cet article sur la délinquance d'hier et de demain, comment conclure, sinon en affirmant qu'il est difficile pour le moment de prédire ce que seront exactement les nouvelles formes de délinquance et l'ensemble du volume de la délinquance, mais il devrait être bientôt possible de les anticiper puisque nous sommes sensibilisés au phénomène et à condition d'être attentifs aux signes avant-coureurs qui apparaîtront. 


\section{ANNEXE \\ Méthode de prévision}

Box et Jenkins (1971) dans leur livre Time series analysis : forecasting and control ont développé des techniques d'analyse de séries chronologiques permettant le traitement de séries statistiques stationnaires ou non stationnaires, ainsi que l'analyse de séries présentant un mouvement saisonnier. Ainsi, ces techniques s'adaptent bien aux données de la délinquance décrite précédemment. La généralité de l'approche de Box et Jenkins justifie amplement l'attention que ces méthodes d'analyse ont reçue dans les écrits. Etant donné que ces techniques sont largement discutées ${ }^{1}$, nous nous bornerons à résumer les étapes d'application de la méthode aux diverses séries qui nous intéressent.

Le modèle général de Box et Jenkins appliqué à une série brute sans composante saisonnière se formule comme suit :

$$
\emptyset_{p}(B)(1-B)^{d} H_{t}=e_{o}+e(B) A_{t}
$$

où $\quad H_{t}$ : série brute non différenciée

$\varpi$ (B)e (B) : polynominiales en $B$ de degré $p$ et $q$ respectivement et dont les racines sont en dehors du cercle unitaire.

1. Le Journal of Royal Statistical Society, (1973) 136, part 3, consacre plus de 50 pages (p.293-351) à la discussion des techniques de Box et Jenkins. Plusieurs références sur la méthode et ses applications y sont mentionnées.
$A_{t}$ : séries de variables aléatoires indépendamment et identiquement distribuées avec comme moyenne 0 et variance $o_{a}^{2}$.
$e_{0}$ : une constante.
d : degré de différenciation de la série.
B : opérateur de retard : $B^{\mathrm{k}} \mathbf{H}_{\mathrm{t}}-\mathrm{H}_{\mathrm{t}-\mathrm{k} \text {. }}$

Ce modèle est appelé un modèle intégré autorégressif à moyenne mobile.

Le problème consiste alors à identifier et à estimer les différents paramètres susceptibles de mieux expliquer le comportement observé de la série $\mathbf{H}_{\mathrm{t}}$ afin de prédire le plus adéquatement possible son comportement futur.

La procédure suivie suppose :

- l'estimation des fonctions d'autocorrélation et d'autocorrélation partielle qui permet l'identification du modèle ;

- le modèle identifié est ensuite ajusté par interactions minimisant la somme des carrés des résidus;

- des tests sont faits pour vérifier la pertinence du modèle. 
TABLEAU 1

Série $\mathrm{H}_{\mathrm{t}}=$ Ensemble de la délinquance $=1932$ à 1972

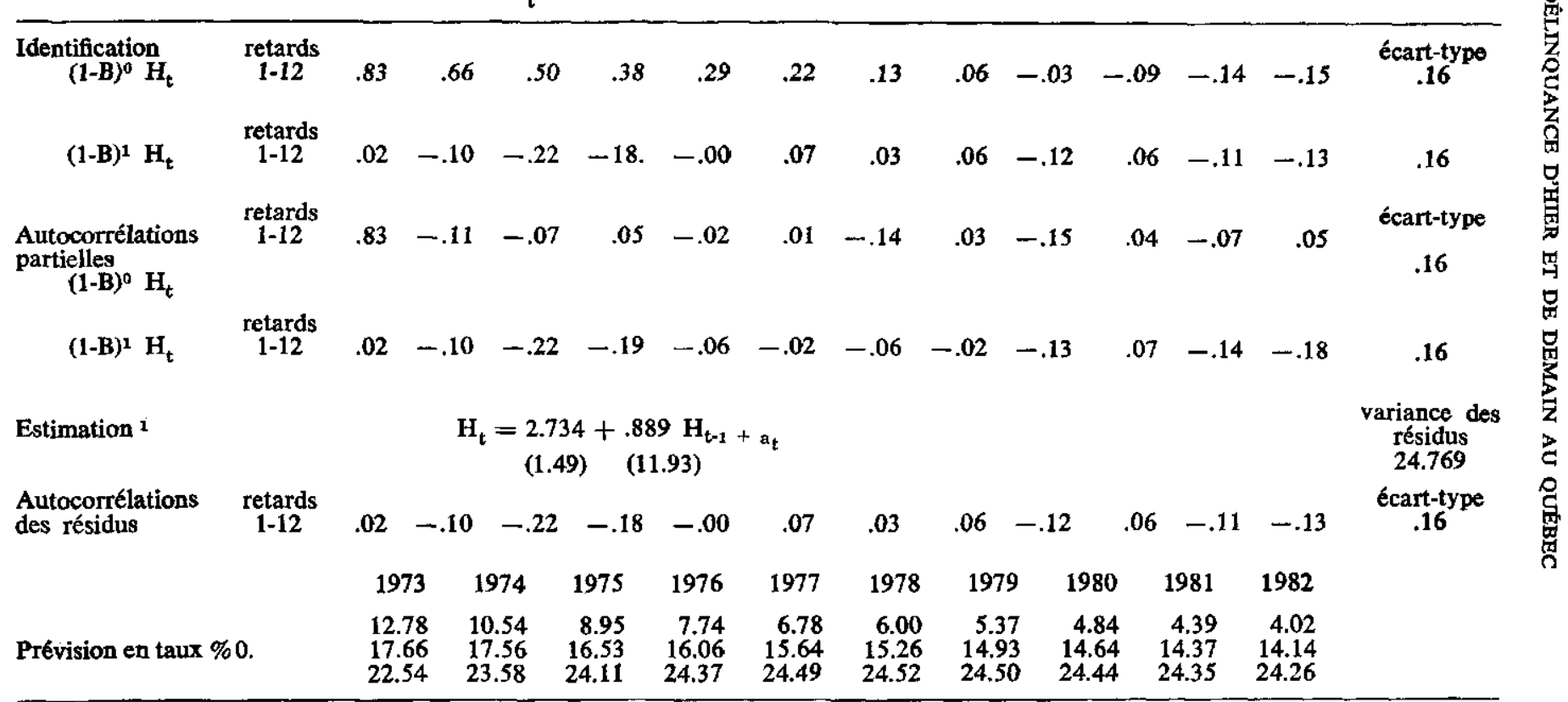

1. La valeur du $t$ statistique est mise entre parenthèse.

2. La première donnée de la fourchette est la plus petite, celle du centre est la prédiction auquel on s'attend et celle du bas est la plus élevée. Cette fouchette a été obtenue avec un coefficient de confiance de $95 \%$.

Une prédiction de taux négatif a été ramenée automatiquement à 0.0 


\section{BIBLIOGRAPHIE}

BOX ET JENKINS (1970) : Time Series Analysis Forecasting and Control, Holden-Day.

BUREAU FEDERAL DE LA STATISTIQUE (1970) : Analyse d'une série chronologique et projection relative aux taux de criminalité, Canada, 1949-1971.

CARLSSON, C. (1968) : Statistik Tidskrifdg, 273, Tioskrifty.

CHEVRIER, P., M. LEBLANC et C. MONTMARQUETTE (1974) : Etude prévisionnelle de la délinquance juvénile à Montréal, Montréal, G.R.I.J.

CHEIN, I. (1963) : Some Epidemiological Vectors of Delinquency and its Control, New York, Research Center for Human Relation.

CHRISTIANSEN, K.O. (1958) : - La criminalité prévue dans les grandes cohortes de naissance ,, Nordisk Tidsskrift for Kriminal Videnskak.

CHRISTIANSEN, K.O. (1967) : The Post-war Trends of Crime in Selected Europeans Countries ", in : The President's Commission on Law Enforcement and Administration of Justice, Washington, U.S. Government Printing Office.

COHEN, J. et A. BLUMSTEIN (1973) : A Theory of Punishment Stability ", The Journal of Criminal Law and Criminology, 64, 2, juin 1973 , p. 198.

COMITE DES PRISONS (1962) : Rapport du comité relatif au développement des prisons de jeunes, Betaenkning, Kobenhava.

CONSEIL DE L'EUROPE (1966) : Quatrième conférence de directeurs d'instituts de recherches criminologiques, Strasbourg, vol. II.

DELAGRAVE, M. (1972) : * La consommation de drogue chez les étudiants du secondaire et du collégial de l'île de Montréal en 1969 et en 1971 \%, Information sur l'alcoolisme et les autres toxicomanies, numéro spécial.

DURKHEIM, E. (1960) : Règles de la méthode sociologique, Paris, P.U.F.

FERRI, E. (1893) : la Sociologie criminelle, traduit de litalien par L. Tessier, Paris, Alcan, III, 640 p.

FRECHETTE, M. (1973) : la Délinquance aujourd'hul et demain, Conférence au Colloque de l'Association des officiers de probation du Québec (10-11 décembre 1973). 
GIBBONS, D.C. (1968) : Delinquent Behavior, New Jersey, Prentice-Hall Sociology Series, 276 p.

GIFFEN, P.J. (1965) : Crime and Its Treatment in Canada, Rates of crime and delinquency, W.T. McGrath, Toronto, p. 59-91.

GILBERT, P.J. (1965) : la Police d Montréal : Etude monographique de la professionnalisation des services de police, thèse de maitrise, Département de criminologie, Université de Montréal, $260 \mathrm{p}$.

GLENNIE, C.M. (1971) : Report of a method of forecasting prison population, Stransbourg, Conseil de l'Europe, ronéo.

HAIJNAL, J. (1954) : The Prospect for Population Forecasts. Comptes rendus du Congrès mondial de la population, Nations-Unies, vol. II.

HENRY et al. (1966) : l'Etude prospective de la criminalite, Conseil de l'Europe, IVème conférence d'instituts des directeurs de recherches criminologiques.

HUBER, M. (1941) : Cours de démographie et de statistique sanitaire, vol. VI, Paris, Herman.

JASINSKI, M.J. (1968) : * La délinquance des mineurs et des jeunes adultes en Pologne de 1951 à 1962 ", in : La délinquance juvénile en Europe, Bruxelles, Editions de l'Institut de Sociologie, p. 45-71.

JEPSEN, J. et L. PAL (1967) : Scandinavian Crime Forecasting Project, Danemark, Conseil de l'Europe, DPC/C D.

JEPSEN, J. et L. PAL (1969) : - Recherches prospectives sur le volume et la structure de criminalité , Etudes relatives d la recherche criminologique, Stransbourg, Conseil de l'Europe.

JOURNAL OF ROYAL STATISTICAL SOCIETY (1973) : 136, part. 3, p. 293-351.

LEBLANC, M. (1968) : la Délinquance juvénile d Montréal : 1960-1968, Montréal, Département de criminologie, Université de Montréal.

LEBLANC, M. (1974) : la Délinquance juvénile au Québec, Montréal, G.R.I.J,

LEBLANC, M. (1974) : Forecasting Juvenile Delinquency, Procecdings of the Second International Symposium on Criminal Justice Information and Statistics Systems, San Francisco.

LEGENDRE, G., R. MENARD et M. LEBLANC (1973) : les Bandes de jeunes : ampleur et nature du phénomène d Montréal, Montrél, G.R.I.J.

LUNDEN, W.A. (1964) : Statistics on Delinquents and Delinquency, Springfield, Thomas.

MARTINEAU, P. (1974) : l'Abandon scolaire, Montréal, G.R.I.J.

MIDDENDORF, W. (1960) : Nouvelles formes de délinquance juvénile : origine, prévention et traitement, New York, Organisation des Nations unies, Département des affaires économiques et sociales.

MINISTERE DE LA JUSTICE DE SUEDE (1970): On the Evolution of Criminality up and Including the Year 1975, Stockholm.

MOGLESTUE, I. (1945) : Crimes, Generations and Economic Growth, Artikler fra Statistik Sentralbrya, Oslo,, 12, 19.

NOTESTEIN, E. et al. (1944) : The Future Population of Europe and the Soviet Union, Genève, Société des Nations.

OGBURN, W.F., J.L. ADAMS et S.C. GILFILLAN (1946) : The Social Affects of Aviation, Boston, Horighton Biffin. 
PINATEL, J. (1963) : Criminologie, t. III du Traité de droit pénal et de criminologie, de P. Bouzat et J. Pinatel, Paris, Dalloz.

PRESIDENT'S COMMISSION ON LAW ENFORCEMENT AND AD. MINISTRATION OF JUSTICE (1967) : The Challenge of Crime in a few Society, Washington, U.S. Government Princing Office.

QUETELET, A. (1835) : Sur l'homme et le développement de ses facultés ou essai de physique sociale, Paris.

RENGBY, (1955), (1958), (1960), (1966) : Les travaux dirigés par S. RENGBY se trouvent exposés in : RENGBY, S., Kungl, proposition, $n^{\circ} 140 / 1955$, p. 147.

RENGBY, S. (1970) : application de pronostics de la délinquance en Suède $*$, in : l'Etude prospective de la criminalité, Strasbourg, Conseil de l'Europe ronéo.

ROBERT, Ph. et Y. CHIROL (1969) : Essai de prévision de la criminalité légale *, Compte général de l'Administration de la justice pour 1967, Paris, Ministère de la Justice.

SCHUESSLER, K. (1971) : * Continuitees in Social Juridiction *, in : Costner, L.H., Sociological Methodology, Jossey Boss, London.

SOROKIN, D.A. (1957) : Social and Cultural Dynamics, Porter Sargent Publisher, Boston.

STATISTIQUE CANADA (1971) : Statistique de la criminalité (police), Ottawa, Information Canada.

TORNUDD, P. (1967) : Prévision de la tendance du nombre de femmes détenues en Finlande, Helsinki, ronéo.

TORNUDD, P. (1969) : Prévision de la tendance de la criminalité, Etudes relatives à la recherche criminologique, Strasbourg, Conseil de l'Europe, 25.

VOY AUBUSSON de CAVARLAY, B., Ph. ROBERT et J. TOISER (1963) : * Recherches prévisionnelles en criminologie ", Futuribles, S.P. 